\title{
Caracterização de recém-nascidos internados em uma unidade canguru da região norte do Brasil
}

\author{
Characterization of newborns admitted to a kangaroo unit in northern Brazil
}

\begin{abstract}
Caracterización de los recién nacidos ingresados en una unidad canguro en el norte de Brasil
\end{abstract}

Cintia Flôres Mutti ${ }^{1}$, Leidiene Ferreira Santos ${ }^{2}$, Bruna Gonçalves dos Santos ${ }^{2}$, Raíra Lopes Amaral de Souza ${ }^{1}$, Eliane Tatsch Neves ${ }^{1}$, Graciela Dutra Senhem ${ }^{1}$, Silvana Bastos Cogo ${ }^{1}$, Aline Cammarano Ribeiro ${ }^{1 *}$.

\section{RESUMO}

Objetivo: Caracterizar os recém-nascidos de uma Unidade de Cuidado Intermediário Neonatal Canguru (UCINca) de um Hospital referência na saúde materno infantil do estado do Tocantins. Métodos: Pesquisa documental, descritiva com abordagem quantitativa. Coleta de dados realizada em registros dos recém nascidos de uma UCINca no período de abril de 2011 a agosto de 2015, a partir os critérios de inclusão e após aprovação do Comitê de Ética em Pesquisa. Resultados: Em relação ao tipo de parto prevaleceu o cirúrgico $65,9 \%$ (310). O peso ao nascimento variou de 700 gramas a menor que $2 \mathrm{~kg}$. A prematuridade $91,5 \%$ (430), outros agravos à saúde foram identificados ao nascimento. Além disso, observou-se o ganho de peso em cada etapa sendo possível afirmar que o método é efetivo em relação ao ganho ponderal de peso do bebê, afirmando seus benefícios. Conclusão: A prematuridade apresentou-se como principal diagnóstico entre os recém-nascidos. Dessa maneira, ressalta-se a importância de um pré-natal de qualidade que possibilite avaliar e intervir precocemente nos possíveis agravos à saúde da mãe e do neonato. E, também propiciou exaltar a importância do Método Canguru e a necessidade da implantação de mais unidades nas maternidades brasileiras.

Palavras-chave: Recém-nascido, Método canguru, Terapia intensiva neonatal.

\section{ABSTRACT}

Objective: To characterize newborns in a Kangaroo Neonatal Intermediate Care Unit (kaNICU) of a reference hospital in maternal and child health in the state of Tocantins. Method: Documentary, descriptive research with a quantitative approach. Data collection carried out in records of newborns from a kaNICU from April 2011 to August 2015, based on the inclusion criteria and after approval by the Research Ethics Committee. Results: In relation to the type of delivery, surgery prevailed $65.9 \%$ (310). Birth weight ranged from 700 grams to less than $2 \mathrm{~kg}$. Prematurity $91.5 \%$ (430), other health problems were identified at birth. In addition, weight gain was observed at each stage, making it possible to state that the method is effective in relation to the baby weight gain, stating its benefits. Conclusion: Prematurity was the main diagnosis among newborns. Thus, the importance of a quality prenatal care is emphasized, which makes it possible to assess and intervene early in the possible health problems of the mother and the newborn. And, it also helped to exalt the importance of the Kangaroo Method and the need to implant more units in Brazilian maternity hospitals.

Key words: Newborn, Kangaroo method, Neonatal intensive care.

1Universidade Federal de Santa Maria (UFSM), Santa Maria - RS. *E-mail: alinecammarano@gmail.com 2Universidade Federal do Tocantins (UFT), Palmas - TO. 


\section{RESUMEN}

Objetivo: Caracterizar a los recién nacidos en una Unidad de Cuidado Intermedio Neonatal Canguro (UCINca) de un hospital de referencia en salud materna e infantil en el estado de Tocantins. Método: Investigación documental, descriptiva con enfoque cuantitativo. Recolección de datos realizada en registros de recién nacidos de un UCINca de abril de 2011 a agosto de 2015, con base en los criterios de inclusión y después de la aprobación del Comité de Ética de Investigación. Resultados: En relación al tipo de parto, prevaleció la cirugía 65.9\% (310). El peso al nacer varió de 700 gramos a menos de $2 \mathrm{~kg}$. La prematuridad $91.5 \%$ (430), se identificaron otros problemas de salud al nacer. Además, se observó aumento de peso en cada etapa, lo que permite afirmar que el método es efectivo en relación con el aumento de peso del bebé, indicando sus beneficios. Conclusión: La prematuridad fue el diagnóstico principal entre los recién nacidos. De esta manera, se enfatiza la importância de una atención prenatal de calidad, lo que permite evaluar e intervenir temprano en los posibles problemas de salud de la madre y del recién nacido. Y también ayudó a exaltar la importancia del Método Canguro y la necesidad de implantar más unidades en las maternidades brasileñas.

Palabras clave: Recién nacido, Método canguro, Cuidados intensivos neonatales.

\section{INTRODUÇÃO}

A taxa de mortalidade infantil no mundo reduziu nos últimos anos, porém com uma pequena redução na mortalidade neonatal que são os óbitos entre zero e 27 dias de vida e sobretudo a mortalidade precoce com óbitos entre zero e seis dias. No Brasil estudo realizado entre 2010 e 2015 observou uma lenta redução da mortalidade neonatal entre zero e 27 dias de vida, ocorreram 96.170 óbitos infantis em sete estados analisados e no Distrito Federal (TEXEIRA J, et al., 2019).

As taxas de mortalidades em todas as regiões do Brasil também refletem esse decréscimo, no entanto, as regiões Norte e Nordeste continuam com as maiores taxas de mortalidade infantil. Em 2010 a taxa de mortalidade neonatal da região Norte foi de 14,6, comparada à taxa nacional de 11,2 (SZWARCWALD CL, et al., 2014). Com isso é importante considerar intervenções de saúde direcionadas a mãe e ao bebê, para reduzir o impacto do índice de morte neonatal (SILVA ACFA, et al., 2019).

Cabe destacar que parte da diminuição dos óbitos infantis é decorrente de um conjunto de procedimentos cada vez mais especializados e o uso das tecnologias, entretanto, mesmo com a disponibilização de equipamentos modernos e de profissionais qualificados, as dificuldades alimentares em especial a amamentação desta população, ainda são motivo de preocupação (BOCCOLIN CS, CARVALHO ML, OLIVEIRA MIC, 2015).

Nesse sentido, no Brasil, várias iniciativas foram desencadeadas em nível governamental e não governamental no intuito de contribuir com a redução da mortalidade infantil. Dentre estas, se destacam o Programa de Humanização no Pré-Natal e Nascimento, o Pacto de Redução da Mortalidade Materna e Neonatal, a criação da Rede Norte-Nordeste de Saúde Perinatal, a Rede Cegonha e a Rede Amamenta Brasil. Além destas, são frequentes as capacitações de profissionais de saúde na estratégia da Atenção Integrada às Doenças Prevalentes na Infância (AIDPI) Neonatal e em Reanimação Neonatal (BRASIL, 2014).

Para tanto a redução da mortalidade neonatal foi assumida como uma das metas para minimizar as desigualdades regionais, dessa forma o Ministério da Saúde com objetivo de contribuir para a mudança de postura dos profissionais e visando a humanização ao recém-nascido, publicou, por meio da portaria no 693 de 5 de julho de 2000, a Norma de Atenção Humanizada ao Recém-nascido de Baixo Peso, o Método Canguru (MC), atualizada em 12 de julho de 2007 pela portaria 1.683 (BRASIL MS, 2013).

Como proposta para melhorar os cuidados prestados ao recém-nascido pré-termo e baixo peso, o Método Canguru foi criado com o objetivo de diminuir a mortalidade neonatal, trazendo benefícios para os recém-nascidos, visando uma assistência humanizada e possibilitando maior vínculo com seus pais. Além 
disso, o método promove o aleitamento materno exclusivo, minimiza a dor dos recém-nascidos que necessitam de procedimentos invasivos, redução do tempo de dias internados e crescente desenvolvimento motor (ZIRPOLI DB, et al., 2019).

O Método Canguru (MC) foi criado na década de 70 em Bogotá na Colômbia, como uma alternativa ao cuidado neonatal convencional, com o intuito de promover o contato pele a pele entre o recém-nascido de baixo peso e a mãe ou outros familiares. A partir desse método, o contato pele a pele passou a ser estimulado não só na UTI, mas no alojamento conjunto de mães e bebês (ELY VHMB, et al., 2017).

Estudos apontam evidências científicas para a eficácia e segurança do MC em termos de mortalidade, infecções precoces, vínculos saudáveis, presença contínua da mãe junto do bebê, garantia calor e leite materno, proporcionando maior estabilidade térmica e alta hospitalar precoce (ZIRPOLI DB, et al., 2019; CHARPAKA N, RUIZA JG, et al., 2017).

O MC é constituído por três etapas, a primeira etapa diz respeito ao acolhimento e orientações fornecidas à família, que inicia antes do parto, com identificação das gestantes de alto risco e orientações sobre os cuidados com a gestação e com bebê. A segunda etapa inicia com a internação da díade mãebebê na Unidade de Cuidado Intermediário Neonatal Canguru (UCINca). Por último a terceira etapa com a alta hospitalar, esta exige acompanhamento ambulatorial criterioso do bebê e de sua família dando continuidade à abordagem biopsicossocial (BRASIL MS, 2013).

Cabe destacar que para ocorrer à efetivação do $M C$, potencializando os benefícios já evidenciados em estudos como Zirpoli DB, et al. (2019); Seidman G, et al. (2015), é necessário que a família conheça a proposta e sua posição no método.

Também é fundamental a qualificação de instituições de saúde, profissionais, na direção de desenvolverem o método de maneira qualificada. Vale destacar que desde 1994, em diversos países da América Latina, inclusive no Brasil, houve treinamentos com equipes de Bogotá, ocorrendo uma importante disseminação de conhecimento (CHARPAKA N, RUIZA JG, et al., 2017).

Diante do cenário das políticas públicas criadas em prol da redução da morbimortalidade neonatal, na qual é influenciada por fatores predisponentes de recém-nascidos de baixo peso, tem-se como objetivo: caracterizar os recém-nascidos de baixo peso internados em uma Unidade de Cuidado Intermediário Neonatal Canguru (UCINca). Na direção de subsidiar a assistência de acordo com as especificidades e prioridades de atenção a esta população.

\section{MÉTODOS}

Trata-se de uma análise documental quantitativa, descritiva de caráter retrospectivo, que foi desenvolvida na UCINca de um hospital materno-infantil, que se localiza no Município de Palmas-TO, no qual é certificado pelo Ministério da Saúde como referência à Saúde Materno-Infantil no estado.

O hospital cenário do estudo possui desde 2002 um banco de leite humano, conquistou o título de Hospital Amigo da Criança, é habilitado e certificado desde 2010 como Centro de Referência no Método Canguru para o Estado. Este estudo foi composto por 470 recém-nascidos internados na UCINca no período de abril de 2011 a agosto de 2015.

Foram incluídos na pesquisa aqueles que participaram da $2^{\circ}$ etapa do $\mathrm{MC}$ e obtiveram os critérios exigidos para participar da terceira etapa do método. Os critérios de exclusão foram: recém-nascidos com peso adequado e/ou normal no momento da admissão, ausência de informações acerca do peso ao nascer e/ou idade gestacional.

A coleta de dados ocorreu no período de julho a setembro de 2015. Os dados foram registrados em um instrumento constituído de informações referentes ao parto, idade gestacional, peso, agravos à saúde ao nascimento, idade, peso no momento da admissão e tempo de internação na unidade. Os dados foram armazenados no programa Microsoft Excel 2010 e submetidos à análise estatística descritiva, exploradas por frequência simples e percentuais. Os resultados foram descritos por meio de tabelas e gráficos. 
Essa pesquisa foi aprovada pelo Comitê de Ética e Pesquisa da Universidade Federal do Tocantins (UFT) sob protocolo no 024/2015de 30 de junho de 2015. E, também desenvolvida de acordo com as diretrizes e normas regulamentadoras da Resolução $n^{\circ} 466$ de dezembro de 2012 do Conselho Nacional de Saúde, que dispõe sobre as normas para pesquisas com seres humanos no Brasil (CONSELHO NACIONAL DE SAÚDE, 2012).

Assim como, também atendeu a Portaria n 796, de 27 de junho de 2014 da Secretária Estadual de Saúde do Estado do Tocantins (SESAU), que estabelece o processo de regulação para prática de estudos nas Unidades de Saúde e Setores de Gestão da SESAU (SECRETÁRIA ESTADUAL DE SAÚDE DO ESTADO DO TOCANTINS, 2014).

\section{RESULTADOS}

Foram analisadas 470 internações de RN no período de abril de 2011 a novembro de 2015 , desses RN, verificou-se que uma quantidade significativa das mães $41,7 \%$ (196) residia no município de Palmas. Este serviço é referência para o Estado do Tocantins e áreas circunvizinhas, como o Sul do Pará, do Maranhão e Bahia.

O referido hospital possui administração estadual, prestando assistência hospitalar e ambulatorial, com nível de atenção de média e alta complexidade. Em relação ao sexo prevaleceu o feminino 56,4 \% (265). A seguir a (Tabela 1), será exposto informações referentes aos dados clínicos dos RN.

Tabela 1 - Variáveis relacionadas ao nascimento de RN participantes do Método Canguru de uma Maternidade de Palmas, TO, Brasil, 2015 (N=470).

\begin{tabular}{lll}
\hline Variável (N) & (\%)
\end{tabular}

Tipo de parto

\begin{tabular}{lcc}
\hline Cirúrgico & 310 & $(65,9)$ \\
Vaginal & 149 & $(31,7)$ \\
Não Registrado & 11 & $(2,4)$ \\
\hline
\end{tabular}

\section{Prematuridade}

\begin{tabular}{lcc}
\hline Sim & 430 & $(91,5)$ \\
Outros & 40 & $(8,5)$ \\
\hline
\end{tabular}

\section{Peso ao nascimento}

\begin{tabular}{lcc}
\hline$<1 \mathrm{Kg}$ & 29 & $(6,17)$ \\
$\geq 1$ e $<1,5 \mathrm{Kg}$ & 150 & $(31,91)$ \\
$\geq 1,5$ e $<2 \mathrm{~kg}$ & 262 & $(55,74)$ \\
Não registrado & 29 & $(6,17)$ \\
\hline Total & - & $\mathbf{1 0 0}$
\end{tabular}

Fonte: Mutti CF, et al., 2020; dados extraídos de um Hospital materno-infantil.

Em relação ao tipo de parto prevaleceu o parto cirúrgico $65,5 \%$ (310), no entanto quase totalidade dos RN nasceram prematuros independente ao tipo de parto. O peso ao nascimento variou, mas é possível visualizar maior registro em $\mathrm{RN} \geq 1,5 \mathrm{e}<2 \mathrm{~kg}$ (262 crianças). Além da prematuridade 91,5\% (430), associados ao baixo peso foram observados outros agravos à saúde ao nascimento, tais como: septicemia, PIG (pequeno para a idade gestacional), anemia e doenças respiratórias. A seguir apresenta-se dados da idade, peso e tempo de internação das crianças na UCINca, na (Tabela 2). 
Tabela 2 - Variáveis relacionadas à idade, peso e tempo de internação dos RN participantes do Método Canguru de uma Maternidade de Palmas, TO, Brasil, 2015 (N=470). (contínua).

\begin{tabular}{lcc}
\hline Variável & $(\mathbf{N})$ & $(\%)$ \\
\hline Idade na internação (2º etapa) & & $-2,8$ \\
Não informado & 13 & $-11,1$ \\
$\leq 7$ dias de vida & 52 & $-37,7$ \\
$>7$ e $\leq 14$ dias de vida & 177 & $-17,2$ \\
$>14$ e $\leq 21$ dias de vida & 81 & $-9,1$ \\
$>21$ e $\leq 28$ dias de vida & 43 & $-22,1$ \\
$\geq 29$ dias de vida & 104 &
\end{tabular}

\section{Peso na internação (2 etapa)}

\begin{tabular}{lcc}
\hline$\geq 1$ e $<1,5 \mathrm{Kg}$ & 169 & $-35,95$ \\
$\geq 1,5$ e $<2 \mathrm{~kg}$ & 278 & $-59,14$ \\
Não Registrado & 23 & $-4,9$ \\
\hline
\end{tabular}

Peso de alta da $2^{\circ}$ etapa (3a etapa)

\begin{tabular}{lcc}
\hline$\geq 1$ e $<1,5 \mathrm{Kg}$ & 1 & $-0,2$ \\
$\geq 1,5$ e $<2 \mathrm{~kg}$ & 118 & $-25,1$ \\
$\geq 2$ e $<2,5 \mathrm{Kg}$ & 184 & $-39,1$ \\
$\geq 2,5 \mathrm{~kg}$ & 12 & $-2,6$ \\
Não registrado & 156 & $-33,2$ \\
\hline
\end{tabular}

Peso na alta da $3^{a}$ etapa

\begin{tabular}{lcc}
\hline$\geq 1$ e $<1,5 \mathrm{Kg}$ & 1 & $-0,2$ \\
$\geq 1,5$ e $<2 \mathrm{~kg}$ & 5 & $-1,1$ \\
$\geq 2$ e $<2,5 \mathrm{Kg}$ & 35 & $-7,4$ \\
$\geq 2,5 \mathrm{~kg}$ & 225 & $-47,9$ \\
Não registrado & 204 & $-43,4$ \\
\hline Tempo de internação & & \\
\hline Não informado & 104 & $-22,1$ \\
Até 7 dias & 88 & $-18,7$ \\
De 8 a 14 dias & 99 & $-21,1$ \\
De 15 a 21 dias & 84 & $-17,9$ \\
de 22 a 28 dias & 50 & $-10,6$ \\
29 dias ou mais & 45 & $-9,6$ \\
\hline Total & - & $\mathbf{1 0 0}$ \\
\hline
\end{tabular}

Fonte: Mutti CF, et al., 2020; dados extraídos de um Hospital materno-infantil.

No momento inicial da terceira etapa do MC, o peso das crianças variou de $1.015 \mathrm{~kg}$ a $3.905 \mathrm{~kg}$, e da alta da terceira etapa, o peso das crianças variou de $1450 \mathrm{~kg}$ a $6660 \mathrm{~kg}$. Observou-se a ausência de informações nos registros, no início da terceira etapa, não foi registrado o peso das crianças em 33,2\% (156) casos e no momento da alta, em $43,4 \%$ (204) casos. Sugere-se que tal situação pode levar ao não retorno do RN (Tabela 2). 


\section{DISCUSSÃO}

O método canguru auxilia na promoção da amamentação materna e no crescimento de prematuros com baixo peso ao nascer. A política que norteia esse método é fundamentada no empoderamento familiar e na alta precoce do prematuro para o acompanhamento ambulatorial (CHARPAKA N, RUIZA JG, et al., 2017; NOBRE, et al., 2017)

Estudo realizado com profissionais da enfermagem reforça a importância desses cuidados para o desenvolvimento infantil dos recém-nascidos e também o quanto é fundamental o processo de educação permanente em serviço na direção de potencializar o desenvolvimento desse método (STELMAK, FREIRE MHS, 2017). Dessa forma é importante conhecer elementos que compõem esse método, pois poderão influenciar na promoção de ganho de pessoa adequado (NOBRE, et al., 2017).

Assim, neste estudo observou-se que em relação a procedência das mães dos RN internados na UCINca, o hospital, no qual é um centro de referência no Método Canguru reconhecido pelo Ministério da Saúde e por ser o único no estado, apresentou uma área de abrangência bastante significativa, totalizando 30 municípios vizinhos. Dessa maneira percebe-se a necessidade de implantar em outras maternidades as UCINca, capacitar equipes multidisciplinares de saúde, com o objetivo de disseminar as práticas de humanização preconizadas por esta metodologia, no sentido de melhorar a eficiência e a eficácia da atenção prestada (COSTA R, et al., 2015).

A prevalência do sexo em recém-nascidos foi feminina com 56,4\% (265), o que converge com estudo realizado que também revelou que os 135 recém-nascidos internados em unidade canguru coletados, 0 sexo que predominou foi o feminino (PENELOPE ET, SCOTT GP, KRISTEN G, 2016). No entanto, em relação a este dado não foram encontrados estudos que fundamentassem relação existente entre sexo, prematuridade e baixo peso.

Os dados registrados em relação ao tipo de parto revelam que o cirúrgico ocorreu em 310 casos e 149 vaginais. Independente do tipo de parto, a experiência que este proporciona é vivenciada por cada mulher de diferentes maneiras, nesse contexto se faz necessário atenção, acolhimento e cuidado, assim também como o vínculo. Dessa maneira, com o intuito de possibilitar humanização nesse momento, cabe aos profissionais tratar cada paciente de forma singular e peculiar levando em consideração as necessidades e o bem estar da parturiente e do RN (COSTA R, et al., 2015).

Em relação a prematuridade e o baixo peso ao nascimento são considerados problemas de saúde perinatal prevalentes no mundo e mais frequente nos países em desenvolvimento. Considerado um problema de saúde pública no Brasil e no mundo devido a sua associação com a morbimortalidade neonatal (MEDEIROS FB, PICCININI CA, 2015). Em relação ao componente neonatal, permanece o desafio de reduzir a taxa de mortalidade, a qual apresenta fatores ligados à atenção à gestação, parto e nascimento (LEAL MC, et al., 2018).

No presente estudo, quase a totalidade de recém-nascidos internados na UCINca eram prematuros $94 \%$ (430). De acordo com alguns estudos constatou-se que cerca de $50 \%$ dos casos de partos prematuros são de caráter espontâneo, de causas desconhecidas e muitas vezes multifatoriais. Muitas causas podem estar associadas à prematuridade, como parto cesáreo, renda mensal baixa, estresse na gestação, assistência pré-natal ausente ou inadequada, as alterações placentárias e excesso de líquido amniótico. Outros fatores incluem: mulheres com extremos de idade, sendo maior incidência em mães mais jovens, infecções maternas, baixa escolaridade (DUARTE MMO, FREIRE EEG, OLIVEIRA JFB, 2015; TABILE PM, TEIXEIRA RM, TOSO G, et al., 2016).

Esse dado denota a importância de implementar políticas públicas que corroborem com efetivas intervenções nas práticas de assistência à gestante durante o pré-natal e parto. Evidências mostram a relação da internação em unidade de terapia neonatal com as características maternas e patologias desenvolvidas no período gestacional (DALLA COSTA LD, et al., 2017).

Estudos mostram que, o adequado acompanhamento pré-natal tem se apresentado como um dos principais fatores de proteção contra a prematuridade, o retardo do crescimento intrauterino, o baixo peso 
ao nascer e aos óbitos neonatais. Além disso, possibilita a identificação de problemas e riscos em tempo oportuno para intervenção (GAIVA MA, MODES PSSA, STEVANATO JM, 2015; GUIMARÃES EAA, et al., 2018).

Nota-se que estas ações associadas a medidas educativas em saúde, são imprescindíveis para a prevenção do parto prematuro haja vista, que são fatores determinantes na incidência e morbimortalidade desses recém-nascidos. Estudo realizado acerca do aconselhamento pré-natal em prematuridade extrema mostrou que os pais queriam aconselhamento o mais breve e mais de uma vez, também relataram ser interessante material de apoio para ajudar a visualizar informações (GEURTZEN R, et al., 2019).

As medidas preventivas adotadas no período neonatal envolvem todos os cuidados neonatais oferecidos ao recém nascido prematuro e não são facilmente estabelecidas, uma vez que a fisiopatologia é complexa e multifatorial. Dessa maneira, se faz necessário o desenvolvimento de uma abordagem interdisciplinar o mais precocemente possível para amenizar ou prevenir sequelas (COSTA R, et al., 2015).

Nesse estudo, além da prematuridade, alguns dos recém-nascidos também apresentou outro tipo de agravo à saúde, como doença respiratória, sepse, pequeno para idade gestacional, anemia e outros. Entende-se, assim, a importância da prevenção de gestações de risco focadas na atenção à saúde da mulher em idade reprodutiva e na assistência qualificada e adequada ao pré-natal, ao parto e ao recémnascido. Sendo, portanto, possíveis de serem modificados com diagnóstico precoce e tratamento adequado, a fim de evitar complicações perinatais e de reduzir o número de óbitos infantis (RYSAVY MA. et al., 2015).

Analisando os dados da tabela 2, o baixo peso foi observado em 95,09\% dos RN internados na unidade apresentando peso entre $1 \mathrm{~kg} \mathrm{e} 2 \mathrm{~kg}$ na admissão. A literatura nos mostra que alguns dos critérios de elegibilidade para permanência do RN na segunda etapa do método canguru é o peso mínimo de $1.250 \mathrm{~g}$, estabilidade clínica e nutrição enteral plena, através de seio materno, sonda gástrica ou copo (BRASIL MS, 2013).

Partindo-se para a análise da influência do nível de escolaridade na realização de parto cesáreo, mães com menos escolaridade, tem mais chances terem recém-nascidos com baixo peso. Esta associação pode estar relacionada ao baixo padrão socioeconômico destas mães, que possivelmente apresentam menor ganho de peso na gestação, início tardio ou baixa qualidade do pré-natal (OLIVEIRA MC, et al., 2015).

Também há relação com desvios nutricionais gestacionais, tanto para a obesidade quanto para o baixo peso, e tais fatores relacionaram-se intrinsecamente ao peso de nascimento dos recém-nascidos. Dessa forma, o acompanhamento nutricional deve preconizar um ganho de peso adequado e gradual, a fim de diminuir os riscos para a mãe e para o bebê (SOUZA DKS, et al., 2015).

A variável peso mostrou no estudo que o ganho ponderal durante a o período de internação na UCINca, ou seja, segunda etapa do método, foi de 1,5 a 2 em 25,1\% (118), de 2 a $2,5 \mathrm{Kg} \mathrm{em} \mathrm{39,1 \%} \mathrm{(184)} \mathrm{dos}$ bebês. O peso mínimo para alta hospitalar do RN internado na enfermaria canguru é $1.600 \mathrm{~g}$ e que o mesmo deve ser acompanhado pela equipe que o assistiu até o alcance do peso de $2.500 \mathrm{~g}$ por meio do seguimento ambulatorial (BRASIL MS, 2013). Estudo mostra que de acordo com o relato de algumas mães, a experiência de vivenciarem o método na segunda etapa traz maior tranquilidade no manuseio com o bebê, explicitada, sobretudo, nos cuidados posteriores à alta hospitalar (OLIVEIRA MC, et al., 2015).

O tempo de internação variou de 7 a 29 dias não sendo informado um total de 104 registros, o que totaliza mais de $20 \%$ dos recém-nascidos internados. Vale ressaltar que o período de internação pode variar de acordo com a evolução de cada díade e a adaptação dos pais aos cuidados que devem ser prestados ao seu filho. A evolução de ambos acontece a cada etapa, importante que ocorra nesse período de internação, visitas frequentes com intervenções (MEDEIROS FB, PICCININI CA, 2015).

A adoção do MC possibilita humanização na assistência, estimulando o vínculo afetivo mãe-filho, melhor adesão ao aleitamento materno, além de propiciar redução nos custos hospitalares. Ressalta-se que tanto no Brasil, como nos países desenvolvidos, o MC é uma proposta para beneficiar a saúde dos recémnascidos, sem substituir a tecnologia hoje utilizada nas unidades neonatais. Também proporciona às mães 
conhecimentos, pois as vivências adquiridas a partir do método permite identificar a diferença entre sono, respiração, temperatura e ganho de peso dos seus filhos (BILOTTI CC, et al., 2016).

A partir dos dados coletados associados à literatura é possível afirmar a contribuição do método com o ganho de peso do bebê em cada etapa do método em relação ao ganho ponderal, afirmando seus benefícios. Além disso, a caracterização dos recém-nascidos possibilitou visualizar variáveis importantes para prevenção da morbimortalidade infantil.

Observou-se como uma limitação do estudo registros incompletos, limitado a ampliação dos dados, sem possibilidade de verificação da terceira etapa do método. Destaca-se que a terceira etapa é fundamental para o acompanhamento do crescimento e desenvolvimento da criança, pois nela ocorre à avaliação pósalta e a capacidade de adaptação dos pais aos cuidados domiciliares.

\section{CONCLUSÃO}

Os resultados do estudo possibilitaram caracterizar os recém-nascidos internados em uma unidade canguru, com isso destaca-se que a prematuridade foi o principal diagnóstico entre os $\mathrm{RN}$, além disso observou-se outros diagnósticos como sepse, doença respiratória e o baixo peso. Dessa maneira, ressaltase a importância de um pré-natal de qualidade que possibilite avaliar e intervir precocemente aos possíveis agravos à saúde da mãe e do neonato. Concluiu-se que a possibilidade de conhecer esta população e adquirir conhecimentos acerca da Metodologia Canguru, permitiu exaltar a importância do método e a necessidade da implantação em mais unidades de maternidades brasileiras, como propósito de uma assistência humanizada e qualificada ao recém-nascido de baixo peso.

\section{REFERÊNCIAS}

1. BILOTTI CC, et al. Método mãe canguru para recém-nascidos de baixo peso: revisão da literatura. Saúde e Pesquisa, Maringá (PR). 2016; 9(3):587-595.

2. BOCCOLIN CS, CARVALHO ML, OLIVEIRA MIC. Fatores associados ao aleitamento materno exclusivo nos primeiros seis meses de vida no Brasil: revisão sistemática. Rev Saúde Pública, 2015; 49(91): 01-16.

3. BOCCOLIN CS, CARVALHO ML, OLIVEIRA MIC. Fatores associados ao aleitamento materno exclusivo nos primeiros seis meses de vida no Brasil: revisão sistemática. Rev Saúde Pública. 2015; 49(91): 01-16.

4. BRASI. Ministério da Saúde. Secretaria de Atenção à Saúde. Manual Técnico de Atenção Humanizado ao Recém Nascido de Baixo Peso Método Canguru [Internet]. Brasília; 2013:204.

5. BRASIL. Ministério da Saúde. Secretaria de Atenção à Saúde. Departamento de Ações Programáticas e Estratégicas. Manual AIDPI neonatal / Ministério da Saúde. Secretaria de Atenção à Saúde. Departamento de Ações Programáticas e Estratégicas, Organização Pan-Americana da Saúde. Coordenação Cavalcante, RS, et al. 5ed. Brasília; Ministério da Saúde; 2014: 228.

6. CHARPAKA N, RUIZA JG. The Kangaroo Mother Care Method: from scientific evidence generated in Colombia to worldwide practice. Journal of Clinical Epidemiology. 2017; 86:125-128.

7. CONSELHO NACIONAL DE SAÚDE. Resolução n. 466, de 12 de dezembro de 2012. Aprova as diretrizes e normas regulamentadoras de pesquisas envolvendo seres humanos [Internet]. Brasília; 2012.

8. COSTA R, et al. Interdisciplinaridade na atenção humanizada ao recém-nascido de baixo-peso num centro de referência nacional do método canguru. Holos. 2015; 3:404-414.

9. DALLA COSTA LD, et al. Fatores preditores para a admissão do recém-nascido na unidade de terapia intensiva neonatal. Rev baiana enferm. 2017; 31(4):e20458.

10. DUARTE MMO, FREIRE EEG, OLIVEIRA JFB. Assistência de Enfermagem à gestante em trabalho de parto prematuro. Revista Interfaces: Humanas e Tecnologia. 2015; 3(1): 01-08.

11. ELY VHMB, et al. Atributos ambientais desejáveis a uma unidade de alojamento conjunto Método Canguru a partir de uma experiência de projeto participativo. Ambient. constr. [online], 2017; 17(2):119-134.

12. GAIVA MA, MODES PSSA, STEVANATO JM. Fatores associados à prematuridade: análise dos dados do sistema de informações sobre nascidos vivos. J Nurs UFPE on line. 2015; 9(12):1110-7.

13. GEURTZEN R, et al. Prenatal counseling in extreme prematurity - Insight into preferences from experienced parents. Patient Education and Counseling. 2019; 102(8):1541-1549.

14. GUIMARÃES EAA, et al. Prevalência e fatores associados à prematuridade em Divinópolis, Minas Gerais, 20082011: análise do Sistema de Informações sobre Nascidos Vivos. Epidemiol. Serv. Saúde. 2018; 26(1): 91-98.

15. LEAL MC, et al. Saúde reprodutiva, materna, neonatal e infantil nos 30 anos do Sistema Único de Saúde (SUS). Ciência \& Saúde Coletiva. 2018; 23(6):1915-1928.

16. MEDEIROS FB, PICCININI CA. Relação pai-bebê no contexto da prematuridade: gestação, internação do bebê e o terceiro mês após a alta hospitalar. Estudo de Psicologia. 2015; 3(32):475-485. 
17. NOBRE R, et al. Weight-Gain Velocity in Newborn Infants Managed with the Kangaroo Method and Associated Variables.Maternal and Child Health Journal. [online]. 2017; 21(1): 128-135.

18. OLIVEIRA MC, et al. Método Canguru: Percepções das Mães que Vivenciam a Segunda Etapa. Revista de Pesquisa Cuidado é Fundamental [Online]. 2015; 7(3): 2939-2948.

19. PENELOPE ET, SCOTT GP, KRISTEN G. The influence of mode of birth on neonatal survival and maternal outcomes at extreme prematurity: a retrospective cohort study. The Australian \& New Zealand journal of obstetrics \& gynaecology. 2016; 56(1): 60-8.

20. RYSAVY MA, et al. Between-hospital variation in treatment and outcomes in extremely preterm infants. New England Journal of Medicine. 2015; 372(19):1801-1811.

21. SEIDMAN G, et al. Barriers and enablers of kangaroo mother care practice: a systematic review. PLoS ONE. 2015; 10(5):01-20.

22. SILVA ACFA, et al. Índice de morte neonatal precoce: Uma análise do perfil materno. REAS/EJCH. 2019; 26: e690.

23. SOUZA DKS, et al. Influência dos desvios nutricionais gestacionais no peso ao nascer de recém-nascidos atendidos pela rede pública de saúde do município de Palmas-TO. Rev Cereus. 2015; 7(1):114-126.

24. STELMAK AP, FREIRE MHS. Aplicabilidade das ações preconizadas pelo método canguru. Revista de Pesquisa:Cuidado é Fundamental Online. 2017; 9(3):795-802.

25. SZWARCWALD CL, et AL. Correction of vital statistics based on a proactive search of deaths and live births; evidence from a study of the North and Northeast regions of Brazil. Popul Health Metro, 2014; 12 (16): 12-16.

26. TABILE PM, et al. Características dos partos pré-termo em hospital de ensino do interior do Sul do Brasil: análise de 6 anos. Revista da AMRIGS. 2016; 60(3):168-172.

27. TEXEIRA JAM, et al. Mortalidade no primeiro dia de vida: tendências, causas de óbito e evitabilidade em oito Unidades da Federação brasileira, entre 2010 e 2015*. Epidemiol. Serv. Saude, 2019; 28(1):e2018132.

28. TOCANTINS. Secretária Estadual de Saúde do Estado do Tocantins. Portaria $n^{\circ} 796$, de 27 de junho de 2014 da Secretária Estadual de Saúde do Estado do Tocantins. 2014.

29. ZIRPOLI DB, et al. Benefícios do Método Canguru: uma Revisão Integrativa. Rev Fund Care Online, 2019; 11 (n.esp):547-554. 